\title{
Typology of Quantifiers and the Mass/Count Distinction: A Case Study of Chinese xie
}

\author{
Kyumin Kim \\ (Cheongju University)
}

\begin{abstract}
Kim, Kyumin. (2017). Typology of Quantifiers and the Mass/Count Distinction: A Case Study of Chinese xie. Language Research, 53.3, 415-443.
\end{abstract}

In a recent approach to the internal structure of nominals, all nouns across languages are proposed to be mass, and thus need to be portioned out (i.e., divided) in order to interact with the count system (Borer 2005): in syntax, division is performed via a Div(ided) head that takes mass noun as a complement, and Div is proposed to be instantiated by an English-type plural $-s$ or a Chinese-type classifier. Once division is performed on a mass, the divided noun can be counted via a numeral that appears in a quantifying phrase (\#P) projected above DivP. Assuming Borer (2005), this paper examines the morpheme xie 'some' in Chinese, and proposes that it is a non-counting quantifier instantiating a \# head that takes DivP as its complement. The proposed account has consequences for the typology of quantifiers and the ongoing debate on the mass/count distinction: division does not necessarily force a counting function, and the mass/count distinction, if it exists at all, is a structural one, not a lexical one.

Keywords: Division, classifier, plural, quantifier, counting

\section{Introduction}

The internal structure of nominals is an intricate product, being the central locus of elements such as definiteness, quantification, plurals, classifiers, gender, and so on. As such, the internal structure of nominal phrases has been under much debate in the literature (e.g, Abney 1987; Ritter 1991; Lyons 1999; Ghomeshi 2000; Borer 2005; Wiltschko 2008; Kramer 2009; among many others). The goal of this paper is to contribute to 
this debate by examining the quantifier xie in Chinese with respect to the syntax of plurals and classifiers, in the framework of the recent proposal on nominal structure in Borer (2005), which has been discussed in recent studies of nominal structure for other languages (e.g., Wiltschko 2008 for Halkomelem (Salish), Mathieu 2012, 2014 for Ojibwe (Algonquian) and Arabic, KM, Kim and Melchin to appear for Korean).

Chinese is a well-known classifier language, and the properties and structure of its classifier phrases have been much discussed in the literature (e.g, Chao 1986; Tang 1990; Chierchia 1995; Cheng and Sybesma 1998; Borer 2005; Cowper and Hall 2012). Although the details differ, recent studies suggest that a Chinese type of classifier is functionally equivalent to an English-type plural $-s$ (e.g., Borer 2005; Cowper and Hall 2012). For instance, in Borer (2005), assuming all nouns across languages are mass, the plural marker $-s$ in English (1a) and a classifier such as ben in Chinese (1b) are proposed to play similar roles, namely portioning out mass, i.e., dividing mass. As such, they are both represented by the same syntactic head Div(ided) as in (2) (to be detailed in section 2).

(1) a. three books

b. san ben shu three CL book 'Three books' (Cheng and Sybesma 1998)

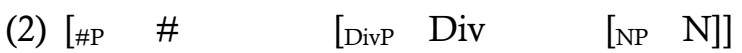

In (2), above DivP is the projection \#P, which is the locus of quantity. For example, the numeral san 'three' appears in this domain. As \#P is a quantity phrase, it can also host quantifiers other than numerals, such as 'a few' or 'much', with different restrictions on the types of complements that it can take, as will be detailed in section 2 .

In this type of nominal structure (2), however, it is not clear how to account for the presence of a plural-like morpheme in a classifier language such as Chinese.1) Chinese has a plural-like morpheme xie, similar to

1) In Chinese, -men is proposed to be a plural morpheme that is suffixed to a noun (e.g., 
English plural $-s$. Following the tradition in the Chinese literature (Iljic 1994; Cheng and Sybesma 2000; Li 1999), I gloss xie as 'some'.2) When this morpheme appears with a noun, the noun is pluralized, as illustrated in (3): xie precedes the noun shu 'book', and the interpretation of the noun is plural 'books'.3) A bare noun in Chinese is well known to be number neutral, allowing either a singular or a plural reading (e.g., Cheng and Sybesma 2000; Rullmann and You 2006). Once xie appears with a bare noun, as in (3), only the plural reading is available. This type of data suggests that xie may be a plural marker, as proposed in Yang (2005) (discussed in section 3.3).

(3) wo lai na xie shu
I come take some book
'I come to take some books (/*a book).'

Adopting Borer's structure (2005), then xie as a plural marker would instantiate Div in (2). However, xie does not allow a numeral larger than one, as shown in (4), which seems to be in contradiction to the proposal of xie as a plural marker that realizes Div.

(4)

*zhe xie san ben shu
This some three CL book
'These three books'

Other studies have proposed that xie is a classifier (e.g., Cheng and

Chao 1968; Norman 1988; Li 1999; KM, Kim and Meng 2017). Thus, the presence of plural -men would not interfere with xie that has to precede the noun. This prediction is correct, as illustrated in (i). This instance is sufficient to show that xie would not compete for the same position as -men, and this paper does not further question the interaction between xie and -men. Note that -men is optional on a noun, and in the data presented in this paper it does not appear.

(i) zhe xie ren-men

This some person-PL

'These people'

2) Chinese has a similar meaning of word to xie, namely dian 'some/little'. However, the distribution of dian is not the same as xie (Iljic 1994). Although interesting, I will leave the investigation of dian as a future research.

3) Chinese data without citation is from consultation with 6 native speakers. 
Sybesma 2000) and at the same time a numeral (Borer 2005). These proposals can be translated into Borer's structure in (2) such that xie realizes Div like a classifier, and moves to \#P; and as such, xie is proposed to be a numeral divider in Borer (2005) (to be detailed in section 3.2). However, this type of analysis suffers from the fact that xie can appear with a classifier such as ge (Iljic 1994; Li 2003a, b), as shown in (5). Given the fact that Chinese disallows the co-occurrence of two different classifiers, ${ }^{4)}$ xie as a classifier, as proposed in Borer (2005), predicts that an example like (5) would be ungrammatical, contrary to the facts. It is also not clear why xie is proposed to be numeral in Borer (2005), as it does not count at all, unlike a standard numeral, such as 'three' for instance.

(5) zhe xie ge shu

that some CL book

'some books'

This paper argues that xie is neither a plural marker like in Yang (2005) nor a classifier/numeral like in Borer (2005). In section 4, it is proposed that xie is a quantifier that originates in \# and it takes DivP as a complement either with a null Div or with an overt Div (instantiated by a classifier $g e$ ). Note that in the proposed account xie does not move from Div, unlike Borer (2005). That is, xie is not a divider. This paper proposes that xie is a quantifier, but it does not have counting function, unlike a numeral that also appears in \#P domain. The proposed analysis of xie has consequences for the typology of quantifiers suggested in Borer (2005), and mass/count distinction much debated in the literature, which will be discussion in section 5 .

4) In Chinese, one of the ways to indicate plurality is reduplication of classifier. See (30) in section 4. 


\section{Nominal Structure: Div(ided) and \#P}

In this section, I discuss the nominal structure proposed in Borer (2005) which is taken as the basic structure to work with in discussing Chinese nominal structure regarding xie. The relevant parts of the nominal structure are illustrated in (6a). In Borer (2005), all nouns across languages are assumed to denote a mass, which needs to be portioned out (i.e., divided) in order for them to interact with the count system. The dividing function is performed by a Div(ided) head (6a). In English, Div is instantiated by the plural marker $-s$, and the noun moves to Div subsequently after the division resulting in plural form cat-s. A noun such as cat in English is assumed to be mass before division, but once it is divided by Div it can be counted, as indicated by a numeral three (6b). In English, a numeral appears in \# (6a), head of the Quantity Phrase, \#P, which plays the role of assigning quantity to a mass or a division of mass. For example, in (6a), a numeral three in \# functions as a counter of the division of mass denoted by cats.

(6) a. $[\# P \quad \#$ three $[$ DivP Div $-s \quad[$ NP cat $]]]$

b. three cats

c. *three cat

When plural $-s$ does not appear, that is, no division of mass is performed, the noun cannot be counted, as the ungrammaticality of (6c) shows. In (6c), the noun is not marked by plural $-s$ which indicates that it is not divided. However, it is counted by the numeral 'three' which results in the ungrammaticality. In the absence of DivP, a mass nominal expression emerges, as illustrated in (7a) with much salt (7b). As the mass is not divided, it cannot be counted by a numeral. However, it can be quantified, as is done by the quantifier much (7b). An ontologically count noun such as cat is also mass when it appears as a bare noun (7c), as assumed, being able to be quantified by the same quantifier as for salt. 
(7) a. [\#P \# much [ NP salt]]]

b. much salt

c. (too) much cat

A noun divided by plural $-s$ can also be quantified by quantifiers (e.g., several or a few) other than numerals, as shown in (8).

(8) a. several boys

b. a few boys

As for these quantifiers in (8), they appear in the same position as a numeral, realizing \#. In Borer (2005), the plural marker $-s$ instantiates the dividing function only, and numerals (e.g., three) and quantifiers of the division of mass (e.g., several/a few) have the counting function. Although a quantifier such as much in (7) also realizes \#, it quantifies a mass that is not divided; thus, no counting function is specified for this type of quantifier.

Turning to the issue of how a singular noun is accounted for under Borer's framework, a singular noun in English is realized with an indefinite article as shown in (9). A singular expression as in (9a) is concluded to be divided since it is counted, i.e., one entity. In Borer (2005), as a bare noun in English is not singular but a mass, Div is absent (see (7a)). However, once a bare noun appears with the indefinite article, it is divided and subsequently counted: the article originates as Div, and moves to \# as in (9b). As such, an indefinite article plays both the dividing and counting functions.

(9) a. a cat

b. [\#P \# $a$ [DivP Div $<a>\quad[\mathrm{NP} c a t]]$

In a classifier language such as Chinese, Borer (2005) proposes that Div is instantiated by a classifier. As shown in many studies (e.g., Tang 1990, and Sybesma 1998, 1999), a classifier in Chinese is needed in order for a noun to be counted.5) Consider the data in (10a-b). The data in (10a) 
indicates that the quantity of shu 'book' is three, as indicated by the numeral san. In this example, the classifier ben for books is present. If the classifier is absent, as shown in (10b), the data becomes ungrammatical. The contrast in the data in (10a) and (10b) indicates that the classifier in Chinese is performing a similar function to the plural marker $-s$ in English, namely the division function. As such, it instantiates a Div head as illustrated in (10c), and a numeral appears in the specifier of \#P.6)

(10) a. san ben shu
three CL book
'Three books'
b. *san shu
three book
Intended meaning:*three books
c. [\#P san\# [DivP Div ben [NP shu $]]$

In sum, in both English and Chinese, a nominal is assumed to be mass, and it needs to be divided in order to interact with the quantifying domain (\#P). In syntax, a Div head projects performing the division function, which is represented by plural $-s$ or a Chinese type classifier. A quantifying phrase, \#P, projected above DivP, can perform counting function. A counter, a numeral, merges in \#P, but other mass quantifiers, without the counting function, can also appear in \#P.

5) Cheng and Sybesma (1998) propose that classifiers in the language can be divided into two different types. However, as with Borer (2005), I assume that this difference does not interfere with the division function of classifiers. See Borer (2005) for detail.

6) Chinese allows a bare classifier without a numeral to occur with a noun. A bare classifier-noun sequence has a singular reading only (Cheng and Sybesma 2000), e.g., ben shu 'a book'/*'books' (see (16a) in section 3.2). Building on this fact, similar to the English definite article $a$ (see (9)), Borer (2005) proposes that a bare classifier in Chinese also realizes a \# head in addition to a Div head. This proposal is extended to classifiers that appear with a numeral; for example, ben in (10c) moves to \# head. Thus, in Borer's structure, (10c) proposed for Chinese, a numeral appears in the specifier of \#P, unlike an English one that appears as the head \# (see (6a)). 


\section{Basic Data on xie and Previous Approaches to xie}

In this section, I first provide the basic distribution of xie, which will be helpful for the rest of the discussion, and then discuss two recent approaches to xie. One is from Borer (2005), who proposes that xie is a dividing cardinal (henceforth, numeral), building on Cheng and Sybesma (1998). The other is Yang (2005), who argues that xie is a plural marker.

\subsection{Basic data on xie}

As mentioned earlier, xie behaves like a plural marker in that it pluralizes the noun that it appears with (e.g., Iljic 1994, Yang 2005), as illustrated in (11). As shown in the data in (11), xie precedes the noun that it pluralizes.

(11) a. wo xian mai xie zhi
I first buy some paper
'I will buy some papers first.'
b. wo lai na xie shu.
I come take some book
'I come to take some books.'

Xie can be preceded by the numeral $y i$ 'one', as shown in (12), but often the numeral is optional as in (11) above. Note that the xie phrase is interpreted as plural even though the numeral means 'one'. Xie phrases can also be preceded by a demonstrative, as shown in (13).7)

$\begin{array}{rll}\text { (12) a. yi } & \text { xie } & \text { zhi } \\ \text { one } & \text { some } & \text { paper }\end{array}$

'Some papers'

7) In Chinese, a demonstrative is viewed as not specified for number (e.g., Iljic 1994, Yang 2005). Thus, in a plural context as in (13), it is interpreted as plural. By contrast, in a singular context as in (i) where xie is absent, it is interpreted as singular. I do not question the syntax of demonstratives in Chinese, and assume that they appear in $\mathrm{D}$ (Li 1999, Yang 2005).

(i) zhe (ge) zhi

This (CL) paper

'this paper' 


\section{b. yi xie xuesheng one some student 'Some students'}

(13) a. zhe yi xie zhi this one some paper 'these papers'

b. zhe yi xie xuesheng this one some student 'these students'

Moreover, xie can be followed by a classifier such as $g e$, as illustrated in (14). As indicated in (14), ge is optional.
(14) a. yi xie (ge) zhi
one some (CL) paper
'Some papers'
b. yi xie (ge) xuesheng
one some (CL) student
'Some students'

In the next two sections, I discuss the recent proposals on xie, and discuss shortcomings of those proposals.

\subsection{Xie as a dividing numeral}

Borer (2005) proposes that xie is a dividing numeral such that it originates in Div as a divider, and it moves to a \# position where an English-type numeral merges (see section 2), as illustrated in (15) for the example xie shu 'some books'.

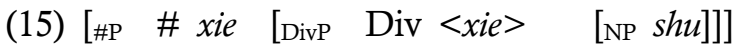

$$
\begin{aligned}
& \text { 'some' 'book' }
\end{aligned}
$$


Following Cheng and Symbesma (1998, 2000), xie is assumed to be a (plural) classifier, and thus it instantiates a Div head. Consider the data in (16) and (17).

(16) wo xiang mai ben shu
I want buy CL book
'I would like to buy a book.'

(17) wo xian mai xie shu
I want buy $C^{\mathrm{pl}}$ book
'I would like to buy some books.'

(Cheng and Sybesma 2000)

In (16), ben is a classifier that appears with a noun such as shu 'book'. As shown in this example, a noun in Chinese is interpreted as singular when it appears with a bare classifier.8) On the other hand, xie, which is proposed to be an indefinite classifier in Cheng and Sybesma (2000), yields a plural reading only, as illustrated in (17). Thus, xie is referred to as a plural classifier $\left(\mathrm{CL}^{\mathrm{p}}\right)$ in Cheng and Sybesma (2000). As the gloss indicates, xie also has a quantity reading 'some'; given this type of quantity reading, Borer (2005) suggests that xie must move to \# from Div.

Under this view, xie plays a dividing role similar to a usual classifier like ben in (16). It also has a quantifying function as its interpretation suggests, and thus it appears in \#. According to Borer (2005), this view captures the fact that xie cannot appear with other numerals, as illustrated in (18a). In (18a), the numeral san 'three' indicates the quantity of the noun shu 'book', but xie is ungrammatical with the numeral. The ungrammaticality contrasts with the grammaticality of the usual classifier ben as in (18b). According to Borer (2005), a numeral that belongs to the quantificational domain is not allowed with $x i e$, as xie also occupies the quantificational domain \#P.

8) In Borer (2005), a bare classifier such as in (16) is analyzed as realizing Div and moves to the \# head, similar to the indefinite article $a$ in English (see (9)). Like an English article, a bare classifier counts a divided noun, giving rise to a singular entity. 


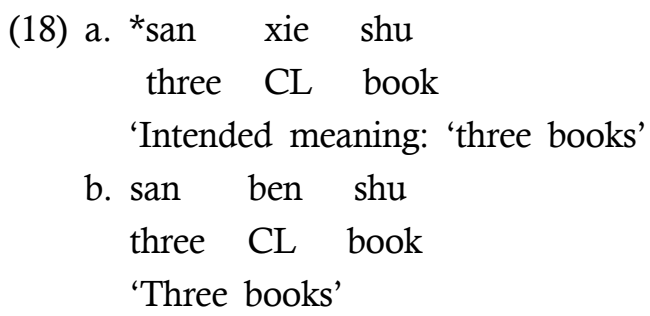

In section 4, I reject Borer's proposal, and propose that xie originates as a \# head that has a specific type of complement, namely DivP (see section 4). In this section, I mention two reasons for the objection. First, the argument that xie and a numeral cannot co-occur as they both appear in the quantification domain \#P does not appear to be sufficient to capture the incompatibility of a numeral and xie in (18a). In Borer's approach, a numeral in Chinese appears in the specifier of \#P (see section 2); for instance, a numeral san in (18a) will appear in the specifier of \#P. In (15), where xie is proposed to end up on a \# head, the specifier of \#P would be available for a numeral; therefore, co-occurrence of numeral and xie would be expected to be grammatical, contrary to fact (18a). Moreover, proposing xie as a numeral seems to be incorrect. As will be shown in section 4, xie cannot be characterized as a numeral, as it does not play the role of counter unlike a usual numeral.9)

\subsection{Xie as Num $[+\mathrm{PL}]$}

Yang (2005) proposed that xie is a plural marker and as such it merges in Num (in the sense of Ritter 1991), as illustrated in (19c):

(19) a. na xie shu
that some book
'those books' *'that book' $^{\text {'th }}$

9) It is not clear why xie is proposed to be a 'numeral' in Borer (2005), as no direct account has been provided; it may be because xie appears in \#P like a numeral, which I do not further question. 
b. na shu
that book
'that book'

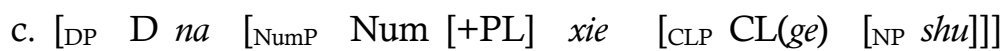

In structure (19c), xie instantiates the [+PL] feature on Num, which yields a plural reading when it occurs with a noun such as shu 'book', as shown in (19a). Yang's (2005) evidence comes from the difference in interpretation when xie is present and absent. As indicated in (19a), a singular reading is not allowed in the presence of xie. In contrast, in the absence of xie, a singular reading is available as in (19b).

Moreover, xie can co-occur with the classifier ge as shown in (20), although ge is optional. As illustrated in (19c), ge instantiates a CL head that appears in a lower position than Num, which captures the compatiblity of xie and the classifier ge. Building on this compatibility, Yang (2005) argues that xie cannot be viewed as a classifier, which is also pointed out in Iljic (1994), unlike Li and Thompson (1989), Cheng and Sybesma (2000), Norman (1988), and Li (2003). When ge is absent, on the other hand, CL is proposed to be semantically vacuous and merely a phonological place holder. Thus, in the proposed structure for xie in (19c), a null $\mathrm{CL}$ is present when $g e$ is absent.

(20) zhe xie (ge) xuesheng
this some CL student
'these students'

The proposal that xie cannot be a classifier head is also supported by the fact that in Chinese two different classifiers are not allowed to co-occur, as shown in (21). Either wei or ge can be used as a classifier for the noun xuesheng 'student', but not both together, as the ungrammaticality of (21) suggests.10) If xie were a classifier, it would be predicted to be ungrammatical to co-occur with another classifier ge, contrary to the fact (20).

10) The classifier wei is a more respectful version than the classifier ge. 


\section{(21) * zhe wei ge xuesheng this CL CL student}

Intended meaning: 'these students' (Adopted from Yang 2005)

In Yang (2005), the [+PL] feature on Num indicates plurality, while CL denotes 'singularities', not 'singular', which is represented by the feature [+SG]. The feature [+SG] refers to a set of atomic individuals, while singular denotes a single entity. In Yang (2005), the feature [+SG] is indicated on Num, despite of indicating the semantics of CL. This is illustrated in the schematic structure $(22 b)$.
a. san ben shu
three CL book
'Three books'
b. [NumP san Num [+SG] [CLP CL ben [NP shu]]] 'three'
'book'

In structure (22b), a numeral appears in the specifier of NumP. According to Yang (2005), a Num head with the [+SG] feature does not interfere with the interpretation of a numeral larger than one. For example, a numeral larger than one such as san 'three' indicates plural, but the plural semantics is compatible with the feature [+SG] on Num, as the grammaticality of the example in (22a) suggests. Although a specific explanation why this is so has not been provided, it seems that the feature [+SG] is not an opposite correspondence to the feature [+PL] instantiated by xie. In other words, the feature [+SG] does not indicate singular as opposed to plural; therefore, $[+\mathrm{SG}]$ on Num seems to be compatible with numeral larger than one such as san, which denotes plural meaning.

However, this view seems to raise incorrect predictions and some confusion. For example, Yang's account for the well-known ungrammaticality regarding xie as shown in (23) seems to be problematic with his proposed structures in (19c) and (22b). In (23), xie appears with the CLP san ben shu 'three books', which is ungrammatical. This data is puzzling in that xie as a plural marker is not compatible with a CLP 
where the numeral is larger than one. According to Yang (2005), the ungrammaticality of (23) is due to having two features on a single head, Num: CLP denotes [+SG] reflected on Num but xie instantiates [+PL] on Num. Num cannot hold two features [+SG] and [+PL] together, and thus the ungrammaticality as in (23) results in.

\section{(23) * na xie san ben shu that some three CL book Intended meaning: 'those three books'}

However, this account of the ungrammaticality seems to pose an internal problem to Yang's (2005) proposal. Yang proposed that the feature [+SG] does not interfere with plural meaning, as discussed above regarding the structure in (22b): a numeral that indicates plural is compatible with the feature [+SG] on Num. More specifically, the feature [+SG], as defined in Yang (2005), does not indicate an opposite value to [+PL]. The feature [+SG] indicates that CLP denotes a set of atomic individuals. In the presence of a numeral, a corresponding number of individual indicated by the numeral can be extracted from the set. Suppose with san 'three' we take 3 individuals from the set denoted by the CLP, which results in a set that consists of atomic sums of three, i.e., plural, and Num in this case has the $[+\mathrm{SG}]$ feature. In this view, the feature $[+\mathrm{SG}]$ on Num is compatible with plural. Thus, it is not clear why the two features, which are not conflict in meaning, cannot co-occur, and the ungrammaticality of (23) remains unexplained.

A more serious problem with Yang's proposal is that no justification has been provided as to why Num, the locus of number, bears either [+ PL] or [+ SG (Singularities)], which are not opposite feature values. In the standard case of Num, Num holds either [+plural] or [-plural] which yields a plural or singular reading of the noun in question. In Yang's proposed structure, it is not clear how singular reading (of a bare noun in Chinese) can be accounted for in the absence of a [-plural]-type feature. Finally, it is not clear why the semantics of CLP should be represented on the Num head. 
In this section, I showed that xie as a dividing numeral as in Borer (2005) and xie as a plural marker instantiating Num head in Yang (2005) are not favored for the reasons discussed for each case. In what follows, I propose that xie is a quantifier without a counting function.

\section{Analysis: Xie as a Non-Counting Quantifier}

In this section, I propose that xie is a quantifier instantiating a \# head but does not have the role of counting, unlike a numeral. Moreover, I propose that xie is neither an English type of plural marker nor a standard classifier in the language. The proposed structure of xie is illustrated in (24).

(24) $[\# \mathrm{P} \quad(y i) \quad \#$ xie $[\operatorname{DivP} \quad \operatorname{Div}(g e) \quad[\mathrm{NP} \quad \mathrm{N}]]]$

Unlike in Borer (2005), xie does not originate as a Div head, as it does not have dividing function, unlike a standard classifier. The first piece of evidence that xie cannot be on a Div head is the fact that it can co-occur with a general classifier ge, as noted in Yang (2005) and also in Iljic (1994) (see (20) in section 3.3). Also, as noted in Yang, Chinese does not allow the co-occurrence of two different classifiers (see (21)). If xie were a classifier, it would be unable to co-occur with ge, which is not the case. Thus, xie cannot be realizing a Div head, instantiated by a classifier in the language. The current proposal that xie cannot be a Div head, which is realized by English plural maker $-s$, suggests that xie cannot be a plural marker, contra Yang (2005).11)

In the proposed structure in (24), xie takes DivP as a complement, and Div can be realized with the classifier $g e$ or can be null. I propose that the semantics of Div in both cases is purely division, devoid of the specific unit information indicated by a usual classifier such as ben, the classifier for the noun 'book', or $z h i$, the classifier for a noun such as $b i$ 'pen'. For instance, ben indicates a unit of volume. As such, it can

11) In fact, xie does not yield the usual plural reading of the noun that it appears with, unlike plural $-s$. Rather, it quantifies a noun as indicated by its meaning 'some'. 
appear with nouns referring to 'book' or 'note', but not with a noun like ren 'person'. The classifier ge is known as a general classifier in that it can appear with a noun without any other classifiers or can be employed as an alternative to another classifier (Iljic 1994). A similar view has been found in experimental studies (Myers et al. 1999a, b). The studies showed that $g e$ is a default classifier lacking lexical semantics, e.g., information on the unit of division proposed in this paper, unlike other standard classifiers in the language. For example, the classifier ben, which denotes a unit of volume, can appear with shu 'book', which can be divided by volume (see (18b)). However, it cannot appear with an object such as 'car' (25a) that cannot be divided by volume. A similar case is shown with classifier liang that indicates a unit of vehicle such as 'car', as illustrated in (25b). Thus, this classifier will not be allowed with a noun that cannot be divided by a unit of vehicle, as shown in (25c).

(25) a. *san ben chezi

three CL car

Intended meaning: 'three cars'

b. san liang chezi

three CL car

'Three cars'

c. *san liang ren

three CL person

Intended meaning: 'Three people'

As for the classifier ge, it does not have this type of semantics. In other words, ge does not indicate the type of unit for the noun that it appears with, as supported by its distribution with a wide range of nouns, as illustrated in (26). As in (26a), it can appear with an abstract notion goujia 'country', with a person ren (26b), or a vehicle chezi 'car' (26c).12)

12) According to a reviewer, the co-occurrence of ge and xie may be more restricted than presented here, being limited to a noun such as ren 'person'. With the speakers that I consulted, the use of ge with xie is allowed not only with ren, but also with other nouns that indicate an object or a thing such as $z h i$ 'paper' (see section 3). Due to the scope of this paper, I leave more thorough investigation of this issue for future research. 


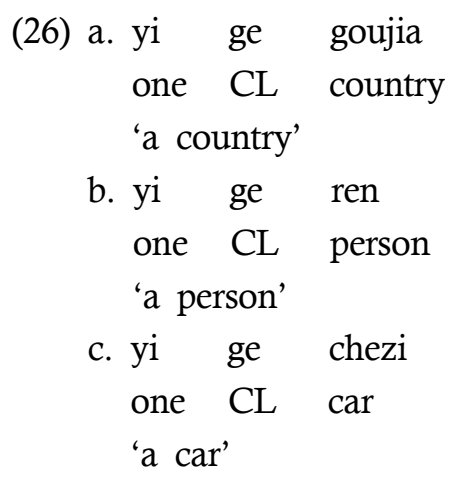

(Myers et al. 1999a)

Lacking the information on the unit, I propose that what is left for classifier ge should be the meaning inherent to a classifier, namely division.

When $g e$ is absent, Div is null but its function as division is intact, similar to when ge is present. This argument is based on Iljic (1994) who suggested that xie appears with a noun phrase whose ontological meaning is countable or discrete. For example, xie is not compatible with a mass noun such as shui 'water' (27), in contrast to its compatibility with a noun ren 'person' that is countable (27b).

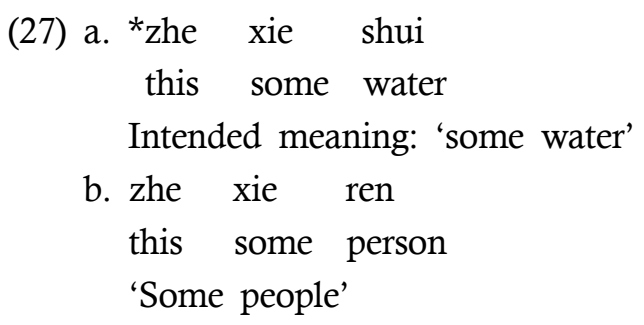

Interestingly, however, xie can have a grammatical reading with a mass noun. For example, (27a) is grammatical when it has an interpretation such as 'different trickles of water' or 'different qualities of water' (Iljic 1994). As these grammatical interpretations suggest, xie, when it appears with a mass noun, results in pluralities that are divisible. I take this fact to suggest that xie takes a nominal complement that is divided.

Implementing this proposal syntactically, I argue that xie as a head takes DivP as a complement, where Div can be null. Without the presence 
of a standard classifier, a null Div has the semantics of a divider devoid of the unit of division, just like when $g e$ is present in Div. In formalizing this type of Div, I assume that the feature [class(ification)] is imposed by a standard classifier, as also proposed in Cowper and Hall (2012). A standard classifier, in addition to division function, also specifies a unit of division, represented by a [class] feature; for example, Div realized by a classifier ben has a [class] feature such as volume.13) In contrast, Div realized by ge or a null Div, the complement of xie, lacks such a feature. This aspect of the proposal captures the fact that in the absence of $g e$, other classifiers are not allowed with xie, as shown in (28). Xie cannot appear with classifiers such as ben (28a) or $z h i(28 b)$. This is because xie takes a DivP complement whose head does not have a [class] feature, but the classifiers in (28) realize Div that has a [class] feature.

(28) a. *zhe xie san ben shu
this some three CL book
'Those three books'
b. *Zhe xie liang zhi bi
this some two CL pen
'Those two pens'

In the proposed account, xie is a non-counter, which is a logical consequence of not instantiating Div. Xie has a DivP complement that does not have information about the unit, which follows from the proposed property of xie, being a non-counter. In a classifier language such as Chinese, for a noun to be counted, a classifier must appear. In the present paper, a classifier instantiates a Div head with [class] feature; thus, xie, as a non-counter, should select Div without [class] feature. Div without a [class] feature denotes a divided nominal, but it does not indicate into what type of unit the nominal is divided. For example, a classifier ben indicates that a nominal that it appears with should be divided into volumes. Thus, when the nominal is counted by a numeral, the nominal

13) For example, this may be represented as [class: volume]. In other words, assuming minimalism (Chomsky 2000), the feature [class] may be valued for a specific unit. 
is counted by volume. In the case of a noun that appears with xie, the noun is divided but without the information on which unit it should be divided into. In the absence of units of division, thus, the noun cannot be counted by unit, disallowing a numeral such as san 'three', as shown in $(29 a)$.

$\begin{array}{lll}\text { (29) a. *san xie } & \text { shu } \\ \text { three some } & \text { book } \\ \text { 'Three books' } & \\ \text { b. (yi) xie } & \text { shu } \\ \text { IND some } & \text { book } \\ \text { 'some books' } & \end{array}$

Thus, the ungrammaticality of (29a) supports the current proposal that xie is a non-counter. I further propose that the xie phrase indicates indeterminate number. Evidence comes from the fact that xie is compatible with an optional indeterminate number marker $y i$, glossed as IND, as shown in (29b). In its standard use, $y i$ is a numeral meaning 'one'. However, it does not mean a numeric value of one when it appears with $x i e$, as indicated in (29b): $y i$ does not contribute singular meaning to the whole phrase. Rather, the interpretation of the quantifying phrase headed by xie is plural in number. In particular, its number is indeterminate, i.e., 'some'. I propose this is why a xie phrase can appear with $y i$, which is proposed to be an indeterminate number marker in Yang (2005). Evidence that $y i$ is an indeterminate number marker comes from reduplicated plural forms in the language. In Chinese, one of the means to pluralize a given nominal is reduplication of classifier, as illustrated in (30). In (30a), the reduplicated form of the classifier zhang appears, and $y i$ precedes the reduplicated form. The meaning of (30a) is plural, not singular, as indicated in the interpretation. Note that plural meaning in (30a) is also possible without $y i$, which is optional just like $y i$ that appears with xie in (29b). Similar to $y i$ that appears with xie (29b), yi that appears with reduplicated classifiers does not provide singular meaning, i.e., it is not a standard numeral that bears the counting function. ${ }^{14)}$ This is 
further supported by the ungrammaticality that results if a numeral larger than one such as san appears in the place of $y i$ with the reduplicated plural forms, as shown in (30b), which is in parallel to the $y i$ that appears with xie (see (29a)). The numeral san 'three' is a counter, and thus it is ungrammatical with reduplicated classifiers, which have no determined number value. ${ }^{15)}$

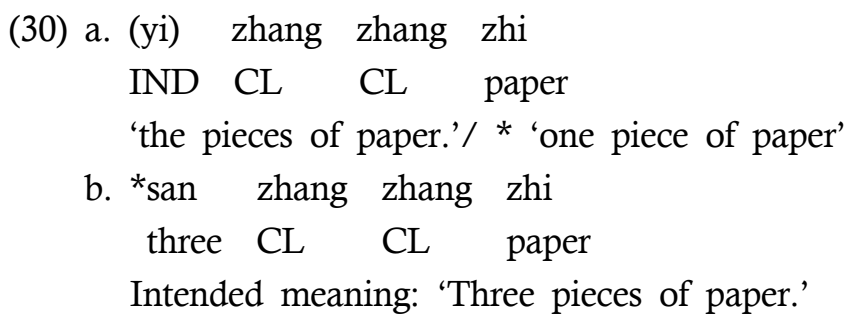

(Yang 2005)

Given the distribution of $y i$ in (30), $y i$ is proposed to be an indeterminate number marker (Yang 2005). As with Yang (2005), I also treat $y i$ in xie quantifier phrases as an indeterminate number marker. Under this view, $y i$ is not a usual numeral such as 'one', and thus $y i$ in (29b) does not have a counting function. This proposal for $y i$ is compatible with the current proposed account of xie as a non-counting quantifier. From this proposal, it follows that as a non-counter, xie does not allow other numerals which have determined numeric values, but allows $y i$, whose number is indeterminate. In syntax, an indeterminate number marker $y i$ merges in the specifier of \#P (see (24)), as it quantifies a noun that it appears with.

14) A reviewer suggested that $y i$ in (30a) maybe used as a numeral 'one'. For example, in (30a), yi zhang zhang could indicate two occurrences of 'one piece' resulting in plural meaning shown in (30a), and in this case, yi may play a role of numeral. It is not clear whether this type of interpretation can hold generally for reduplicated classifiers in Chinese. However, what is clear is that $y i$ in a reduplicated classifier context as in (30a) cannot be syntactically numeral, as it does not share the same syntactic properties as a numeral $y i$ 'one' (Yang 2005). Thus, $y i$ in an example such as in (30a) may not function as a numeral.

15) For the purpose of the paper, I do not pursue the question of how to analyze reduplicated classifiers, but see Yang (2005) for details. 


\section{Consequences}

In this section, I focus on two consequences emerging from the current proposal for xie. One is a consequence of the analysis for the typology of \# in a nominal structure, and the other is an implication for the mass/count distinction, which is much debated in the literature.

\subsection{Situating xie in a typology of quantifiers (\#)}

Under the proposed account, a xie-type quantifier is not found in the typology of quantifiers (\#) proposed in Borer (2005). In particular, in her system, DivP is necessary in order to count (\#P) but not vice versa. In order to count, e.g., via a numeral, a nominal expression has to be divided: structurally, this relation is represented such that \#P takes a DivP complement, as exemplified in (31a). However, \#P does not always require DivP, as illustrated in (31b): \#P can take a mass nominal without division as a complement. In this case, \#P quantifies the nominal rather than counting it.
(31)
a. [\#P \# [DivP Div [NP $\quad$ N]] $]$
b. $[\# P \quad \# \quad[N P \quad N]]$

These two types of quantifiers - counting (31a) and non-counting (31b) - are found in English, as shown in Table 1 below. In this table, the typology of English \# discussed in Borer is summarized with the addition of Chinese classifiers and xie. ${ }^{16)}$

16) In Borer (2005), quantifiers are classified with two dimensions, $[ \pm$ counter] and $[ \pm$ divider], and more quantifiers (from other languages as well) than those presented here are discussed. For the purpose of the paper, I discuss relevant quantifiers only. What is important to the current discussion is that a xie-type quantifier is not found in Borer's typology. 
Table 1. Typology of \# in Borer (2005) and Chinese xie

\begin{tabular}{|c|c|c|c|c|}
\hline & Types of \# & \multicolumn{2}{|c|}{ DivP complement } & $\begin{array}{l}\text { Counting } \\
\text { function }\end{array}$ \\
\hline \multirow{4}{*}{ English } & (i) numeral & Yes & \multirow{3}{*}{$\begin{array}{c}\text { Div } \\
\text { - Instantiated by } \\
-s\end{array}$} & yes \\
\hline & (ii) a few type & Yes & & yes \\
\hline & (iii) indefinite $a$ & Yes & & yes \\
\hline & (iv) much type & No & N/A & no \\
\hline \multirow{2}{*}{ Chinese } & (v) numeral & Yes & $\begin{array}{c}\text { Div [class] } \\
\text { - Instantiated by a } \\
\text { classifier }\end{array}$ & yes \\
\hline & (vi) xie type & Yes & $\begin{array}{c}\text { Div } \\
\text { - optionally } \\
\text { instantiated by } g e\end{array}$ & No \\
\hline
\end{tabular}

Numeral (i), a few-type (ii), and indefinite marker a-type (iii) quantifiers belong to the set of counting quantifier \#s as figured in (31a), while much-type (iv) quantifiers belongs to the set of non-counting quantifier \#s as shown in (31b).17) For instance, in a structure such as a few boys, \#P has a counting function when \# is merged with a few. As indicated in (ii), this \# requires DivP: compare a few boys vs. *a few boy. In order for a few (\#) to count, DivP realized by plural $-s$ should appear as the complement of a few (\#). On the other hand, with much as in much salt, \#P does not have counting function and thus no DivP is required (iv).18) In this case, DivP should not be projected, as the ungrammaticality of such projection (realization of plural -s) suggests as in *much salts. It should be noted that Div in English-type languages does not have specific information on the division of unit, unlike Div in the Chinese type that is instantiated by a usual classifier: Div in an English-type plural, and Div [class] in a Chinese-type classifier.

In Borer's typology, the Chinese xie type of quantifier is not attested. Xie does not have a counting function, like the quantifier much (iv).19)

17) The difference between (i)/(ii) and (iii) is that the indefinite marker $a$ in (iii) plays the roles of both division and counting. See section 2 for details.

18) As with much, \#P is projected as it quantifies a nominal expression that it appears with. The counting function is one of quantifying functions expressed by \#P.

19) The proposed typology regarding xie would be further supported by the presence of non-counting quantifier in other languages, which I leave for future research. For 
Unlike much, however, it requires a division of mass to quantify, namely a DivP complement, similar to the quantifier a few or a numeral. Language internally, xie is similar to a usual numeral (v) in that it appears in \#P domain; however, it requires a DivP stripped of specific class information (32a) (similar to an English-type DivP (32b)), unlike a numeral in the language, which requires a DivP with class information (32c).

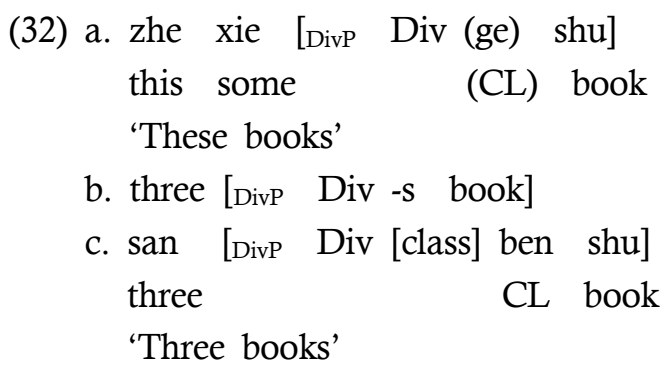

It may seem surprising that even in a classifier language such as Chinese, a DivP without any class information similar to an English-type DivP is attested. However, Div as proposed in Borer (2005), in the absence of a [class] feature, is expected to have only a single function, namely division. ${ }^{20)}$

\subsection{Count and mass distinction and non-counting quantifier xie}

Pushing further the claim that a Chinese-type noun is mass (Chierchia 1995), Borer (2005) proposes that all nouns across languages are mass. A syntactic consequence of this is that all nouns need to be portioned

$-t u l$ in Korean, it may be difficult to be grouped together with $x i e$, as -tul shows different syntactic properties from those of xie. For example, in a recent study, -tul is proposed to be a plural marker that adjoins to a $n \mathrm{P}$ as a modifier (Kim and Melchin to appear). As such, unlike xie, plural -tul is predicted to co-occur with a classifier, which is the case in the language (Kim and Melchin to appear).

20) In recent studies (Mathieu 2012, 2014), it has been shown that the content (i.e., 'flavor' in Mathieu 2012) of Div may vary across languages, and these different flavors of Div are realized with different types of plural. It has been argued that the English-type plural $-s$ and Chinese-type standard numeral classifiers are different 'flavors' or contents that Div can take. Under this view, a [class] feature may be one of the contents that Div can have. 
out (i.e., divided) in order to interact with the count system. Under this view, the mass/count distinction should be understood at the structural level, not at the lexical level, as argued in Borer (2005). As for count, it is a structural product: Div is projected over a mass expression, and counted via the projection of quantifying head \# (33a).21) As for mass, it is lacking the Div projection, although it can be quantified (e.g., much salt) (33b).22)

(33) a. count structure

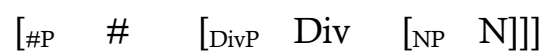

b. mass structure

$[\# \mathrm{P} \quad \# \quad[\mathrm{NP} \quad \mathrm{N}]]$

This view on the mass/count distinction appears to be in contrast to the proposal in Cheng and Sybesma $(1998,2000)$ proposed for Chinese. They argued that in Chinese the mass/count distinction is encoded at the lexical level, which is also pointed out by Borer (2005). In Cheng and Sybesma (1998), mass nouns are selected by a classifier referred to as a massifier, while count nouns are selected by a classifier referred to as a count-classifier. These classifiers are illustrated in (34a) and (34b) respectively. In (34a), ping 'bottle' is a massifier which creates measure for a mass nominal such as jiu 'liquor'. In (34b), on the other hand, $z h i$ is a count classifier which merely names the unit of the noun that it appears with. The noun $b i$ 'pen' in (34b) is ontologically a count noun, and there is a natural unit to count pens. In this case, the count classifier $z h i$ just names this unit, and does not create any measure for the noun.

(34)
a. san ping jiu
three CL.bottle liquor
'three bottles of liquor

21) When \#P is not present in a structure such as in (33a), but a DP projects above DivP, the resulting structure is that of bare plurals in English (Borer 2005). As this is not directly relevant to the purpose of this paper, I do not discuss this issue.

22) Note that mass structure in (33b) is one of the ways in which mass can surface; for example, without \#P, (33b) is still mass. 
b. san zhi bi
three CL pen
'three pens'
(Cheng and Sybesma 1998)

They further propose that count-classifiers make a noun countable as they single out one countable discrete unit. However, massifiers do not make a noun countable, but instead with the addition of massifiers the noun is still a mass. This is because in Cheng and Sybesma (1998), a massifier is considered not to single out a discrete unit of the noun.23) Consequently, the mass/count distinction is visible at the lexical level such that different types of classifier are selected by an ontologically mass or count noun.

However, the proposed account of xie in this paper supports the structural mass/count distinction advocated in Borer (2005), rather than the lexical view in Cheng and Sybesma (1998). The quantifier xie selects a discrete noun represented by DivP, where Div is realized either by $g e$ or by a null morpheme. This suggests that xie takes a structurally count complement. It cannot be viewed that it selects ontologically count nouns, as evidenced by the fact that it can occur with a mass noun such as 'water' as mentioned earlier. When xie appears with a mass noun, the noun has a particular interpretation, as illustrated in (35).

(35) zhe xie shui
this some water
'these different trickles of water'

(Iljic 1994)

The compatibility with mass nouns is not limited to 'water', but this compatibility is productive, as illustrated in (36). Importantly, the interpretations of these examples are count, i.e., divided, not mass, as indicated in the translation, which provides support for the proposed analysis of xie in this paper. For instance, in (36a), xie appears with the mass noun 'snow'. However, this phrase is understood as individualized snow; for

23) No further explanation has been provided for this proposal. Presumably, this may be because a unit denoted by a massifier is still mass in Cheng and Sybesma's view. For example, one bottle of liquor taken as a whole is still mass. 
example, (36a) can be understood in a context where a man brought some snow taken from different mountains, and presented them into different containers respectively.24) The phrase cannot be understood as a whole in a context such as 'these snows fell on to me' where 'these snows' is taken as a pile of snow.

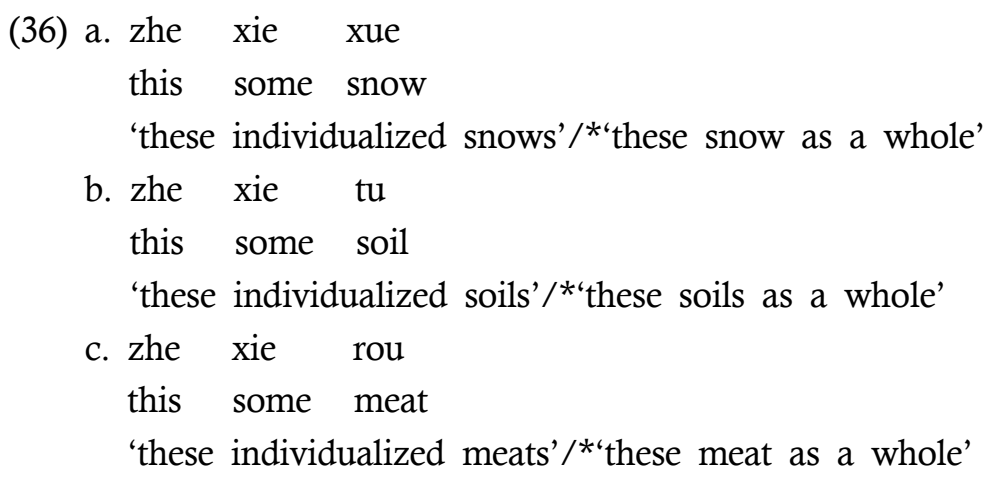

These data show that xie can appear with lexically either a count or a mass noun, but it has to take a structurally count phrase, namely DivP. This suggests that xie is inert with respect to the lexical mass/count distinction, but it distinguishes a structural count phrase from structural mass phrase. Thus, the proposed account supports the structural view of the mass/count distinction, not the lexical one.

24) A reviewer pointed out that $d u o$ 'all' can appear with examples such as in (36), but not with gege 'every'. Consider the following example in (i) where (36a) is embedded in a sentence. As shown in (i), duo is grammatical to appear; however, gege cannot appear in the position of duo. The reviewer suggested that the grammaticality with duo, but not with gege, may indicate that a xie phrase may not strictly denote individuals, unlike the proposal made in this paper.

(i) zhe xie xue dou /*gege hua le

This some snow all /every melt ASP

'These snows are all melted.'

However, settling this issue seems to be less straightforward than it appears. For example, duo cannot appear in a nominal context; thus, *zhe xie xue dou (intended meaning: 'all these snows') is ungrammatical. Moreover, as indicated in the gloss in (i), duo is interpreted as a modifier of event denoted by the verb phrase, rather than of the nominal subject. As pursuing this issue goes beyond the scope of this paper, I leave it for further research. 


\section{Conclusion}

This paper examined the distribution of the quantifier xie in Chinese in the perspective of the recent proposed nominal structure in Borer (2005). Contrary to the previous approaches to xie, it is shown that xie can be neither an English-type plural morpheme nor a standard classifier in the language, both of which are proposed to be instantiated by Div in Borer (2005).

The provided evidence in this paper supports the current analysis of $x i e$ as a non-counting quantifier. More specifically, it is demonstrated that xie cannot be a classifier, and thus it cannot realize a Div head. Also, xie is shown to take a divided mass as a complement, namely DivP. The type of DivP that appears with xie is lacking the information on the unit of division, and formally this type of Div is proposed to lack a [class] feature. This aspect of the proposed analysis suggests that Div in the absence of a [class] feature performs its pure function, i.e., division. Another important consequence of the current proposal for xie is the support for the view that the mass/count distinction, if it exists at all, should be encoded structurally rather than lexically, thereby contributing to the ongoing discussion on the count/mass distinction in the nominal domain (e.g., Chierchia 1998; Krifka 1995; Borer 2005; Doetjes 2012; Bale and Coon 2014).

\section{References}

Abney, Steven. (1987). The English Noun Phrase in Its Sentential Aspect. Doctoral dissertation, MIT.

Bale, Alan and Jessica Coon. (2014). Classifiers Are for Numerals, Not for Nouns: Consequences for the Mass/ Count Distinction. Linguistic Inquiry 45, 695-707.

Borer, Hagit. (2005). Structuring sense, vol. I: In name only. Oxford University Press. Chao, Yuen-Ren. (1968). A Grammar of Spoken Chinese, University of California Press.

Chomsky, Noam. (2000). Minimalist inquiries: The framework. In Roger Martin, 
David Michaels and Juan Uriagereka (eds.), Step by step: Essays on Minimalist syntax in honor of Howard Lasnik, 89-155. Cambridge, MA: MT Press.

Cheng, Lisa Lai-Shen and Rint Sybesma. (1998). Yi-wan tang, yi-ge tang. Classifiers and massifiers. Tsing-Hua Journal of Chinese Studies 28, 385-412.

(1999). Bare and not-so-bare nouns and the structure of NP. Linguistic Inquiry 30, 509-542.

Chinese. Ms. HIL/Leiden University. (2000). Classifiers in four varieties of

Chierchia, Gennaro. (1995). Plurality of mass nouns and the notion of "semantic parameter". Ms., University of Milan.

Cowper, Elizabeth and Daniel Currie Hall. (2012). Aspects of individuation. In Diane Massam, ed., Count and mass across languages, 27-53. Oxford University Press.

Doetjes, Jenny. (2012). Count/Mass distinctions across languages. In Claudia Maienborn. Klaus von Heusinger. and Paul Portner, ed., Semantics: An international handbook of natural language meaning 3, 2559-2580. Mouton de Gruyter.

Ghomeshi, Jila. (2003). Plural marking, indefiniteness, and the noun phrase. Studia Linguistica 57.2, 47-74.

Kim, Kyumin, and Xiao Meng. (2017). Variation in the syntax of plural markers: the case from plural -men in Mandarin. Ms. Cheongju University and Liaocheng University.

Kim, Kyumin, and Paul Melchin. (to appear). Modifying plurals, classifiers, and co-occurrence: the case of Korean. Glossa: A Journal of General Linguistics.

Kramer, Ruth. (2009). Definite markers, phi-features, and agreement: A morphosyntactic investigation of the Amharic DP. Santa Cruz: University of California dissertation.

Krifka, Manfred. (1995). Common nouns: A contrastive analysis of English and Chinese. In Gregory N. Carlson and Francis Jeffry Pelletier, ed., The generic book, 398-411. University of Chicago Press.

Iljic, Robert. (1994). Quantification in Mandarin Chinese: Two markers of plurality. Linguistics 32, 91-116.

Lyons, Christopher. (1999). Definiteness. Cambridge University Press.

Li, Charles and Sandra Thompson. (1981). Mandarin Chinese: A Functional Reference Grammar, University of California Press, Berkeley.

Li, Audrey Yen-Hui. (1999). Plurality in a classifier language. Journal of East Asian Linguistics 8, 75-99.

(2003a). NP as Argument. In A. Li and A. Simpson, eds., Functional Structure(s), Form and Interpretation. Routledge.

(2003b). Making Sense of Language Differences. Journal of 
the Linguistic Association of the Southwest 22, 23-49.

Mathieu, Eric. (2012). Flavors of division. Linguistic Inquiry 43, 650-679. (2014). Many a plural. In Ana Aguilar-Guevara, Bert Le Bruyn. and Joost Zwarts, eds., Weak Referentiality, 157-182. John Benjamins publishing company.

Myers, James, Shu-Ping Gong, and Zhong-Gang Shen. (1999a). The Semantic Content of the General Classifier in Mandarin. Paper presented at the International Association of Chinese Linguistics Eighth Annual Conference. Melbourne, Australia.

Myers, J., C-C Chiang, and S-P Gong. (1999b). Rules vs. Analogy in Mandarin Classifier Selection, ms., National Chung Cheng University; to appear in Language and Linguistics 1.

Norman, Jerry. (1988). Chinese, Cambridge University Press.

Ritter, Elizabeth. (1991). Two Functional Categories in Noun Phrases: Evidence from Modern Hebrew, In Susan Rothstein, ed., Syntax and Semantics 25: Perspectives on Phrase Structure, 37-62. Academic Press.

Rullmann, Hotze and Aili You. (2006). General number and the semantics and pragmatics of indefinite bare nouns in Mandarin Chinese. In Klaus von Heusinger. and Ken P. Turner, eds., Where Semantics Meets Pragmatics, 175-196. Elsevier.

Tang, C.-C. Jane. (1990). Chinese phrase structure and the extended X'-theory. Doctoral dissertation, Cornell University, Ithaca, N.Y.

Wiltschko, Martina. (2008). The syntax of non-inflectional plural marking. Natural Language and Linguistic Theory 26, 639-694.

Yang, Henrietta Shu-Fen. (2005). Plurality and Modification in Mandarin Nominal Phrases. $\mathrm{PhD}$ dissertation. University of Texas at Austin.

Kyumin Kim

Cheongju University

Department of English Language and Literature 298,

Daeseong-ro, Cheongwon-gu Cheongjusi Chungcheongbukdo South Korea

E-mail: kyumin@cju.ac.kr

Received: October 24, 2017

Revised version received: November 26, 2017

Accepted: December 6, 2017 
